potential of a probe did not disturb the space-charge conditions near the other probes even when the magnetic field was applied.

Contrary to the results of Cobine and Gallagher ${ }^{5}$, a sharp maximum of noise was found when the probe potential crossed the local potential in all the experiments which have been carried out

The Cobine and Gallagher hypothesis and the J. J. Thomson-Langmuir theory of electron oscillations in ionized gases do not seem sufficient to explain the results of these experiments.

A complete account of the present work will appear in due course in the Revue Scientifique ${ }^{6}$.

Laboratoire de Physique,

École Normale Supérieure,

45 Rue d'Ulm, Paris, V. Aug. 28.

1 Thonemann, P. C., and King, R. B., Nature, 158, 21 (1946).

2 Denisse, J., and Steinberg, J.-L., C.R. Acad. Sci., 224, 546 (1947) ${ }^{3}$ Greeves. F. D., and Johnston, J. E. McF., Phil. Mag.. 21. 659 (1936). - Spencer-Smith, J. L, Phil. Mag., 19. 806 (1935).

Sobine and Gallagher, J. App. Phys., 18, 110 (1947).

' Steinberg, J.-L., [Rev. Sci, 85, 601 (1947)].

\section{Gyro-interaction of Radio-Waves Obtained by the Pulse Method}

IN March and April 1946, during fourteon nights, experiments were made by the Institute of Technical Physics of the University of Naples in association with the Institut Elettrotecnico Nazionale of Turin, the Vatican Radio and Italian Navy and Air Force, in order to verify if there is a resonance effect in the interaction of radio-waves (gyro-interaction) according with V. A. Bailey's suggestion of $1937^{1}$.

For this purpose a method devised by me was used, consisting in varying the frequency of the disturbing station around the local gyromagnetic frequency, to obtain the maximum of the phenomenon. As soon as the maximum was revealed at the receiving apparatus, the frequency of the disturbing station which has produced the maximum was measured by means of a frequency-meter. This measured frequency is very close to the local gyromagnetic frequency. By doing this, it was possible with a power of 700-800 watts to measure the local gyromagnetic frequency $\Omega=e H / 2 \pi m$, and consequently the value of the total terrestrial magnetic field $H$ in the ionosphere, and also to ascertain a regular variation of the gyro-frequency during the night.

In order to verify the results of those experiments, further investigations were made between January and July 1947, in association with the Italian Navy. The military stations of Augusta (630 m., $1 \mathrm{~kW}$.) and Taranto (1,250 m., $1 \mathrm{~kW}$.) operated as emitters, and Venice, La Spezia, Leghorn and Roma (Sant' Alessandro) as receiving stations. Naples, the wavelength of which varied from 265 to 285 m., operated as disturbing station.

In order to obviate the lack of power of the Naples station (400-500 watts), which, in the first experiments, made observation of the phenomenon very difficult, the pulse-method was applied to this station. The experiments made using the pulse-method have confirmed my suggestion, because, although the power was not much varied, it is believed that the transient is more efficient than a continuous wave in the interaction.

The gyro-interaction was observed frequently, sometimes during a whole hour of transmission, and very easily, notwithstanding the remarkable strength of the atmospheric static. The number of the observations (about forty) obtained in Venice, and often also in Rome, is sufficient to confirm clearly the gyrointeraction. The phenomenon was never observed in Leghorn and La Spezia, perhaps on account of the fact that the rellexion points of the waves of the emitting stations were too far from the 'sky' of Naples. The wave-lengths of the disturbing station which have caused the gyro-interaction are included between 265 and 282 metres. The variation law of the disturbing station frequency was 4 by 4,2 by 2 , and very often I by 1 metre. The behaviour of the phenomenon was generally similar to that in 1946 during the three hours of transmission. While in the first hour the gyro-frequency was low, about 282 metres, in the second hour it became higher, about 272 metres or less, and in the third hour about 265 metres. In 1946 and 1947 , the interaction was stronger between 0200 and 0300 local time, and less easily observable between 0400 and 0500 .

The experiments are being continued with more powerful stations.

Institute of Technical Physics,

Mario Cutolo University, Naples. Aug. 8.

1 Cutolo, M., Carlevaro, M., and Gherghi, M., Alta Frequenza, 111 (Giugno, 1946). Bailey, V. A., Nature, 139, 68 (1937); 139, 838 (1037). Cutolo, M., Ric. Sei. e Ricostr., 1835 (Dic, 1946).

\section{Relativity of Accelerated Motion}

According to the general theory of relativity, accelerated motion is relative; that, however, is difficult to reconcile with experimental evidence; for example, in the case of rotation.

The relativity of accelerated motion follows from the general covarianey of physical laws; thus, if $T_{a}{ }^{0}=0$ (for all four components) represents a physical law in one system of co-ordinates, then in any other system the law is

$$
T_{\mu}=\frac{\partial x_{\alpha}^{0}}{\partial x_{\mu}} T_{\alpha}^{0}=0 ;
$$

that is, it preserves its form.

Laws of physics can therefore give us no information about the system of co-ordinates used; and, since four-dimensional curvilinear co-ordinates correspond to accelerated motion, the latter is purely relative. This difficulty can be explained as follows.

Equation (1) presupposes that (a) there is one to one correspondence between the two spaces, (b) the transformations $\partial x_{\alpha}{ }^{0} / \partial x_{\mu}$ remain finite throughout the space; that, however, need not be the case in a transformation between a Galilean and accelerated spaces.

Accelerated motion is equivalent to a gravitational space, and we should expect the latter to have an impenetrable boundary, since otherwise a particle might acquire in it an infinite velocity. For example, in the case of Einstein's gravitational space, the boundary is $r=2 \mathrm{~m}$. There is, therefore, no one to one correspondence between a Galilean and accelerated spaces.

If a Galilean space can be transformed into an accelerated one, we should expect that on the boundary the transformations $\partial x_{\alpha}{ }^{0} / \partial x_{\mu}$ would become infinite, and (1) would break down. Unfortunately, there are no solutions for accelerated spaces, except in the case of a uniform accelerated motion ${ }^{1}$. 